\title{
Representative Feature Points Detection on Periapical X-ray Images
}

\author{
Chia-Yen Lee, ${ }^{1}$ Liang-Hsin Wang, ${ }^{1}$ Yu-Hsien Lin, ${ }^{2}$ and Chih-Chia Huang ${ }^{3 *}$ \\ ${ }^{1}$ Department of Electrical Engineering, National United University, \\ No. 2, Lien-Da, Nan-Shih Li, Miao-Li 36063, Taiwan R.O.C. \\ ${ }^{2}$ Department of Electronic Engineering, National United University, \\ No. 2, Lien-Da, Nan-Shih Li, Miao-Li 36063, Taiwan R.O.C. \\ ${ }^{3}$ Department of Dentistry, Cardinal Tien Hospital, \\ No. 15, Chezi Rd., Xindian Dist., New Taipei City 23155, Taiwan R.O.C
}

(Received January 18, 2018; accepted August 15, 2018)

Keywords: periapical X-ray images, feature detection, endodontic treatment, Barnard algorithm, alveolar bone

Trabecular bone density below the root tip is often used as a basis for determining treatment efficacy. Before comparing the trabeculae at different time points, it is necessary to register longitudinal images to identify the same location of before and after treatment. In order to achieve accurate image registration, it is important to identify representative feature points in the image. Current feature point detection algorithms are unable to detect representative feature points in periapical X-ray images. The algorithm proposed in this study is based on the conventional Barnard feature point detection algorithm to solve the problem regarding excessive quantity and aggregation of feature points for periapical X-ray images with poor contrast. The results of this study showed that the proposed algorithm can successfully identify representative feature points that are uniformly distributed in periapical X-ray images. This algorithm can be used in the future for longitudinal periapical X-ray image registration and to assist dentists in finding the same location of treatment to determine the efficacy of endodontic treatment.

\section{Introduction}

Odontogenic infection, like dental caries, causes teeth periapical bone infection. Patients who suffer toothache with chronic apical inflammation or dental alveolar abscess feel discomfort when biting. In such cases, endodontic treatment is necessary. The dentist will assess the degree of recovery of these teeth through clinical signs and symptoms, and trabecular bone regrowth observed in periapical radiographic images. For example, bacteria from root canal may migrate into the periapical bone. These cause cortical and trabecular bone destruction. The periapical radiographic image showed black shadows below the root apex [lower first molar (36) mesial root apex radiolucency image indicated by red arrows in Fig. 1(a)]. After surgical root canal therapy and follow over the next year, the area of radiolucency disappears

*Corresponding author: e-mail: frank554@ms13.hinet.net

https://doi.org/10.18494/SAM.2018.2036 


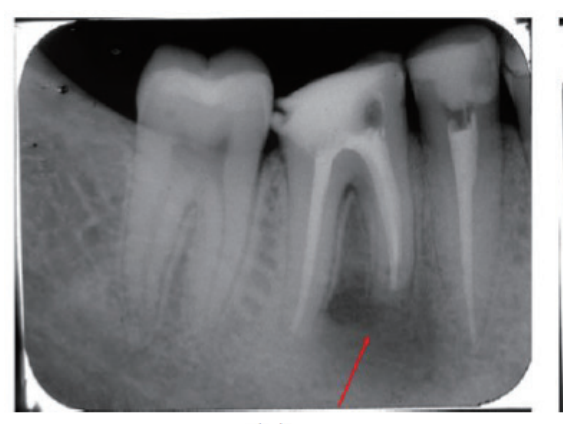

(a)

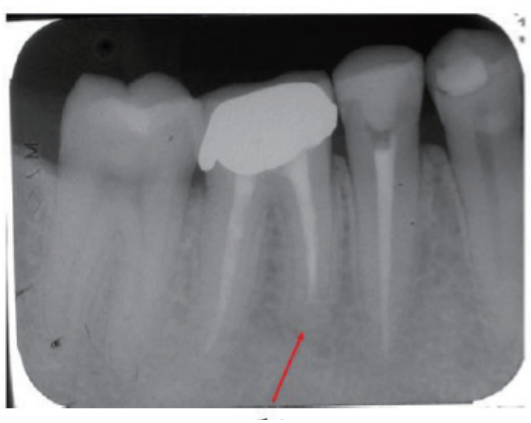

(b)

Fig. 1. (Color online) Periapical X-ray images (red arrows indicate regions of periapical lesions). (a) Before and (b) after treatment.

[as shown in 36 of Fig. 1(b)]. The dentist will determine the efficacy of the endodontic treatment and the degree of recovery of 36 on the basis of the trabecular bone appearance in the radiographic image. At present, the determination of trabecular recovery to a certain extent is considerably dependent on the dentist's experience. However, the root tip positions in X-ray images before and after treatment are not completely identical, as seen in Fig. 2. This makes it difficult to determine the trabecular bone recovery status.

Some researchers have developed algorithms for the quantitative assessment of trabecular bone recovery. ${ }^{(1,2)}$ These methods require the dentist to manually select images at different time points for the same position before conducting quantitative assessment and analysis before and after treatment. However, visualization by the naked eye, followed by manual selection of X-ray images at different time points for the same position is inaccurate. Therefore, it is necessary to perform registration of periapical X-ray images at multiple time points.

In image registration, the results of feature point detection will affect the quality of registration. Therefore, identifying representative feature points of sufficient quantity is important. However, as the gray-scale contrast of periapical X-ray images is not distinct, it is difficult to obtain good feature point detection results. Hence, developing the most suitable detection method for periapical X-ray images is indispensable.

In medical image processing, various different feature point detection algorithms have been developed for image registration. Depending on the detection method, feature point detection algorithms can be classified into four main types: edge detection, corner detection, regional feature point detection, and ridge detection. ${ }^{(3)}$ Edge detection uses the edge pixels of two types of regional images as feature edges. This type of detection includes methods such as Canny edge detection ${ }^{(4)}$ and Sobel edge detection. ${ }^{(5)}$ It is difficult to apply the feature edges obtained from edge detection in image registration; therefore, these methods are usually not used. Corner detection and regional feature point detection can detect representative and robust feature points on images. Corners are the most intuitive features and are the best for showing strong two-dimensional intensity changes that can be clearly differentiated from neighboring points. Corners also have high information content on image position representation. This 


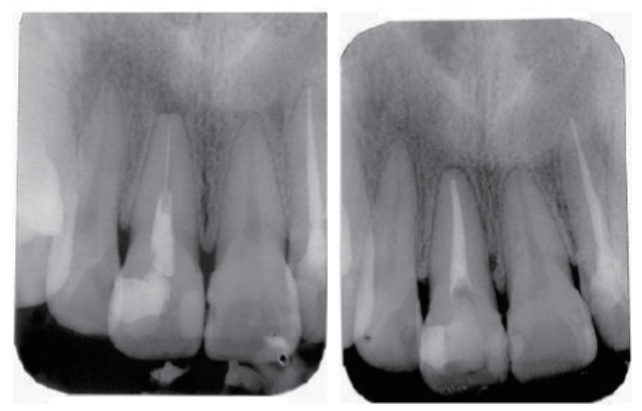

(a)

(b)

Fig. 2. Periapical lesions (a) before and (b) after treatment.

form of detection can more accurately detect the positions of feature points in images with a lot of noise. The algorithm used is also more robust. Thus, several studies are being conducted in this area using methods such as the difference of Gaussian (DoG) feature point detection, ${ }^{(6,7)}$ Harris corner detection, ${ }^{(8-10)}$ and Barnard edge detection. ${ }^{(11,12)}$ Ridge detection is commonly used in distinguishing blood vessels, but it is difficult to detect ridge points in gray-scale images. Therefore, ridge detection is not suitable for use in this study.

Some of the above-mentioned algorithms are more commonly used than others, such as Harris corner detection and DoG feature point detection. The Harris corner detector was developed from the Moravec corner detection algorithm with improvements made by Harris et al. A window is moved around an image, and intensity changes are obtained and used to determine corners. The DoG feature point detection uses a Gaussian filter to produce multiscale spaces. Then, extreme points can be detected from differences in images as feature points. These two detection methods require multiscale images for the analysis of the positions of feature points. However, the corner points of periapical X-ray images are not obvious and the degree of contrast is low. If preprocessing of images is not performed, multiscale analysis will be unable to generate sufficient feature points from the image. The Barnard feature point detection algorithm (Barnard algorithm), a form of the point detection method, can effectively solve the problem of insufficient feature points. The Barnard algorithm uses the gray-scale variance of neighboring pixels as a basis for determining feature points. Pixels with large variances are viewed as feature points. However, problems such as excessive quantity of feature points and uneven distribution still exist when searching for feature points. To reduce the excessive quantity of feature points generated by the Barnard algorithm that are also overly aggregated, the simple use of gray-scale responses to remove unnecessary feature points ${ }^{(13)}$ is not sufficiently effective. In view of this problem, Song et al. developed a modified Barnard algorithm. Song et al. used the cumulative gray-scale percentage to suppress the quantity and distribution of feature points. They used eight directions for screening neighboring feature points and extracted points with higher gray-scale values as feature points. Although this method solves the problem of excessive quantity and aggregation of feature points, its mask is small and requires manual adjustment, which is time-consuming. 
In this study, we developed an improved feature point detection algorithm based on the Barnard algorithm. This method can overcome the problem of insufficient feature points owing to poor contrast of X-ray images and avoids the aggregation of feature points. We also propose a form of the adaptive mask-size selection method, which can decrease the overall detection time.

\section{Materials and Methods}

This study was approved by the Institutional Review Boards of Cardinal Tien Hospital. All the experimental methods were carried out in accordance with the approved guidelines. Written informed consent was obtained from all patients involved in this study.

The proposed feature point detection algorithm was applied to detect the feature points of periapical X-ray images acquired from 35 subjects receiving endodontic treatment.

In this study, we developed a feature point detection algorithm suitable for periapical X-ray images based on the Barnard algorithm in order to identify a sufficient number of representative feature points for use in subsequent image registration. The procedures involved are divided into two steps. In the first step, the conventional Barnard algorithm is used for the initial selection of feature points. This method will produce problems, such as excessive quantity and aggregation of feature points. The second step involves using the aggregated feature points as a basis for extracting extreme gray-scale values of feature points. Screening is performed by regional detection methods. This is done to solve the problem of excessive quantity and aggregation of feature points. Last, the degree of uniformity and radius suppression were used to analyze the effectiveness of this feature point detection method.

Figure 3 shows the flowchart of the modified Barnard algorithm developed in this study. The conventional Barnard algorithm was used for the detection of initial feature points. Regional four-directional smoothing was performed on every pixel in the original image $f$ using Eqs. (1)-(5) of the Barnard functions to obtain four smoothed parameters for each pixel. The lowest smoothed parameter was used as a new value for each pixel.

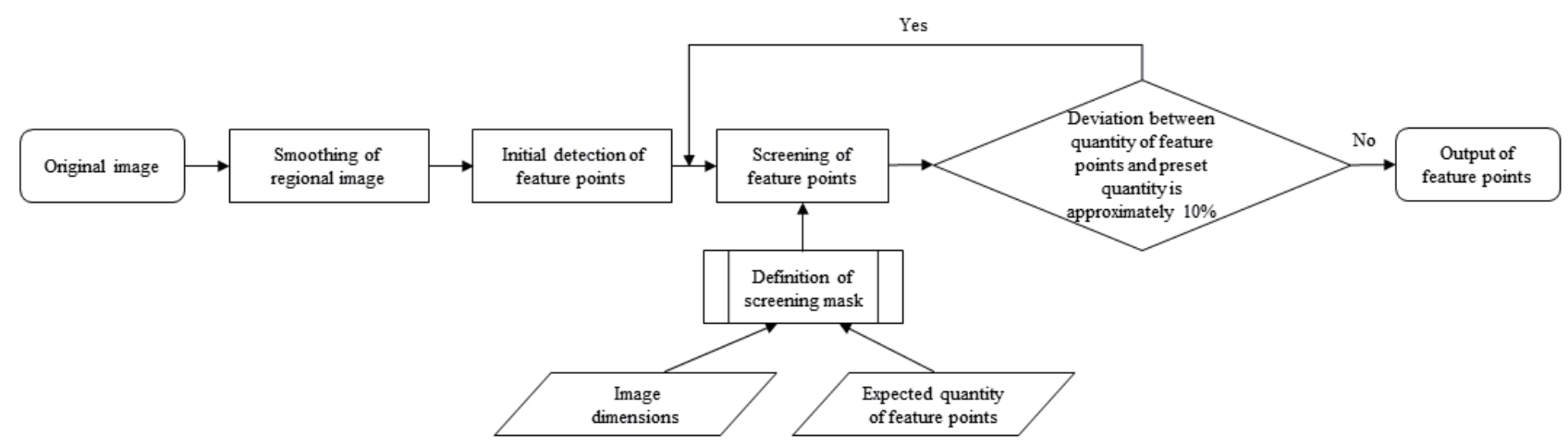

Fig. 3. Flowchart of the proposed algorithm. 


$$
\begin{gathered}
H=[f(m, n)-f(m-1, n)]^{2}+[f(m, n)-f(m+1, n)]^{2} \\
V=[f(m, n)-f(m, n-1)]^{2}+[f(m, n)-f(m, n+1)]^{2} \\
L=[f(m, n)-f(m+1, n-1)]^{2}+[f(m, n)-f(m-1, n+1)]^{2} \\
R=[f(m, n)-f(m+1, n+1)]^{2}+[f(m, n)-f(m-1, n-1)]^{2} \\
t(m, n)=\min \{H, V, L, R\}
\end{gathered}
$$

In these formulas, $(m, n)$ are the pixel coordinates; $H, V, L$, and $R$ are the smoothed parameters; and $t$ is the new pixel value. The mask $w$ was used for the selection of a new image matrix. The maximum value in the mask $w$ was obtained, and the distinctive point was used as a feature point, as shown in

$$
(x, y) \in \max _{(m, n) \in w}\{t(m, n)\}
$$

where $(x, y)$ are the coordinates of the feature point.

Song et al. proposed an improvement of the original Barnard algorithm because of the excessive quantity and aggregation of feature points. The quantity of feature points in their method is affected by the mask size, and multiple rounds of manual adjustment of the mask are required. However, this process is time-consuming.

$$
p=\sqrt{(m \times n) \div N}
$$

In Eq. (7), $p$ is the mask size after the operation, $m \times n$ is the size of the image, and $N$ is the quantity of customized eigenvalues. The preset quantity of feature points was used for adjusting the mask size for screening neighboring feature points in the region. The feature points with the maximum new pixel values were extracted as final feature points, as shown in

$$
\left(x^{\prime}, y^{\prime}\right) \in \max _{(x, y) \in p^{2}}\{t(x, y)\},
$$

where $\left(x^{\prime}, y^{\prime}\right)$ are the coordinates of the final feature point. If the quantity of the final feature points is much lower than that of the customized feature points, the $p$ value is decreased and vice versa. Thus, a quantity of feature points that is similar to that of customized feature points can be generated to achieve the optimal distribution of feature points. 


\section{Results and Discussion}

Figure 4 shows the results of this study. The identified feature points possess the following characteristics: representativeness, sufficient quantity, and uniform distribution within the image. We compared this algorithm with the DoG algorithm, the original Barnard algorithm, and Song's modified Barnard algorithm.

The images in rows 1-5 in Fig. 4 are the original image and the images obtained using the DoG algorithm, original Barnard algorithm, Song's modified Barnard algorithm, and the improved algorithm developed in this study, respectively. From these results, we can clearly see that the feature points obtained using the DoG and Song's modified Barnard algorithms are not uniformly distributed, resulting in excessive aggregation of feature points.

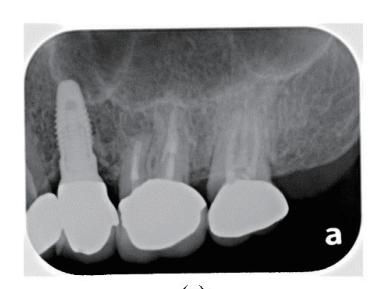

(a)

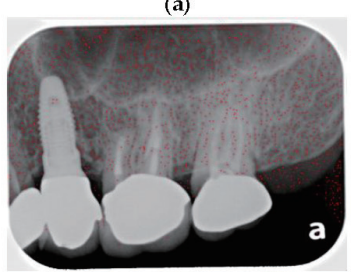

(d)

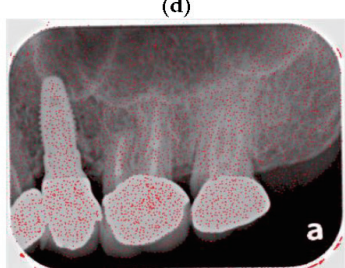

(g)

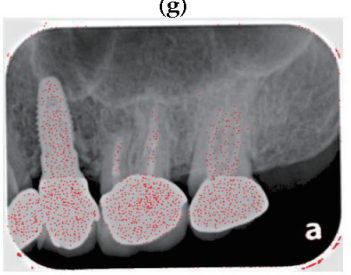

(j)

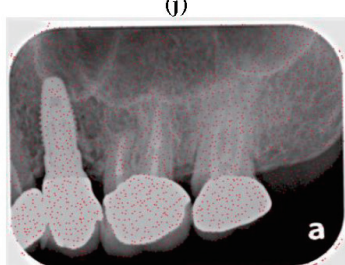

(m)

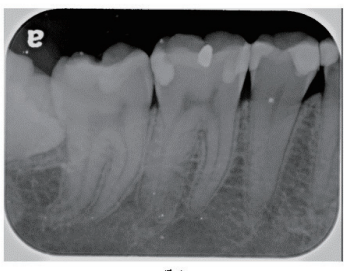

(b)

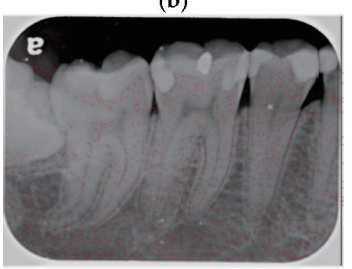

(e)

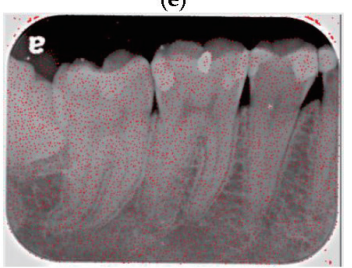

(h)

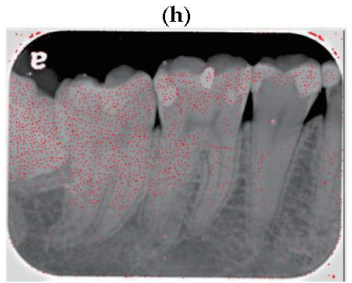

(k)

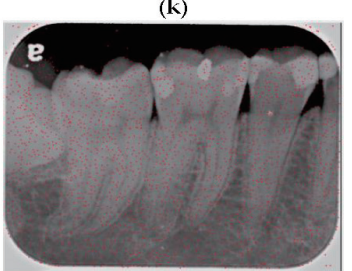

(n)

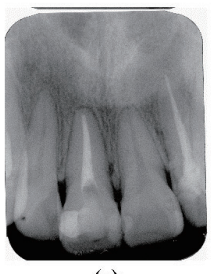

(c)

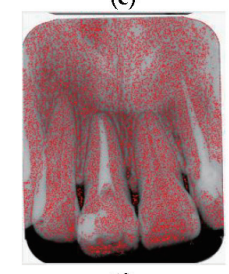

(f)

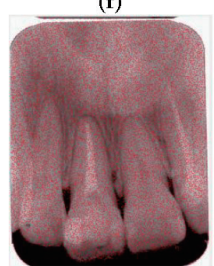

(i)

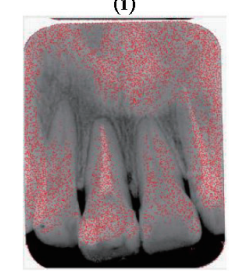

(1)

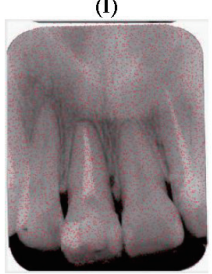

(o)

Fig. 4. (Color online) Detection results using the proposed algorithm and three other algorithms on three different cases. 
In order to verify the effectiveness of the proposed algorithm, the original image Fig. 4(a) was used as a reference for further analysis of the results using degree of uniformity and radius suppression.

\subsection{Analysis of degree of uniformity}

The degree of uniformity was analyzed using a circle with a fixed enlargement size to detect the quantity of feature points and observe whether there was a stable increase in the quantity of detection results to determine if the feature points were uniformly distributed in the image. Table 1 shows the quantity detection results obtained with a detection radius of 10 for interval expansion detection.

Figure 5 shows the analysis curves of the degree of uniformity. The closer a curve is to a straight line, the higher the degree of uniformity. From Fig. 5, we can see that Song's modified Barnard algorithm shows the highest degree of uniformity within the detection range; however, from Fig. 4(j), we can see that the feature points obtained using this algorithm exhibit excessive aggregation and do not show uniform distribution. Compared with the other three methods, the curve of the improved Barnard algorithm developed in this study has the gentlest gradient and thus the highest degree of uniformity.

\subsection{Analysis of radius suppression}

Radius suppression analysis utilizes radius suppression to exclude feature points by calculating the eigenvalues inside a circle since only feature points have eigenvalues. Only feature points with the largest eigenvalues are retained. A higher proportion of excluded feature

Table 1

Analysis results of degree of uniformity.

\begin{tabular}{lrrrrrrrrrr}
\hline & \multicolumn{10}{c}{ No. of detected points } \\
\cline { 2 - 11 } Method & \multicolumn{10}{c}{ Radius (pixels) } \\
\cline { 2 - 11 } & 10 & 20 & 30 & 40 & 50 & 60 & 70 & 80 & 90 & 100 \\
\hline DoG & 3 & 3 & 5 & 9 & 20 & 32 & 51 & 74 & 94 \\
Original Barnard & 14 & 26 & 46 & 67 & 96 & 131 & 179 & 227 & 287 \\
Song's modified Barnard & 1 & 7 & 15 & 27 & 39 & 55 & 80 & 107 & 129 & 165 \\
Proposed improved Barnard & 1 & 9 & 20 & 35 & 45 & 52 & 59 & 68 & 75 & 83 \\
\hline
\end{tabular}

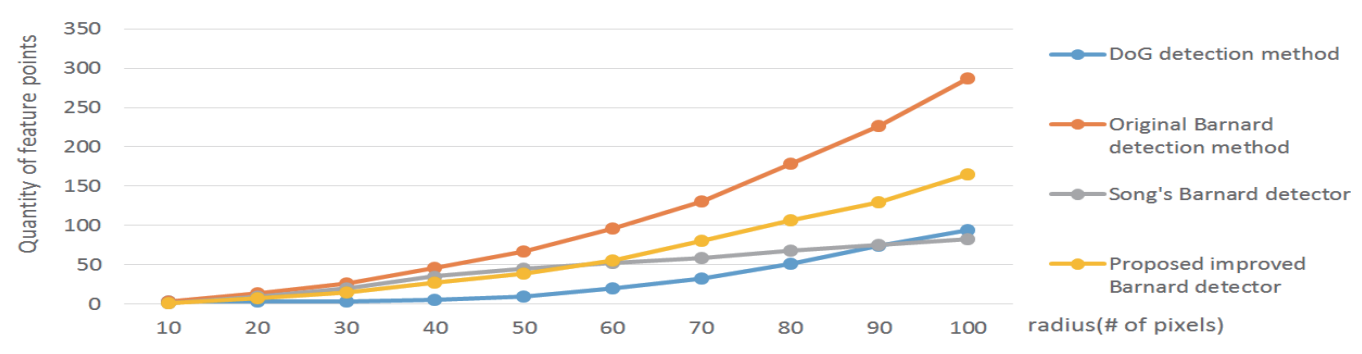

Fig. 5. (Color online) Analysis curves of degree of uniformity. 
Table 2

Analysis of suppression radius.

\begin{tabular}{lccc}
\hline Method & $\begin{array}{c}\text { No. of } \\
\text { feature points }\end{array}$ & $\begin{array}{c}\text { No. of excluded } \\
\text { feature points }\end{array}$ & Exclusion ratio \\
\hline DoG & 1334 & 109 & 0.0817 \\
Original Barnard & 4330 & 876 & 0.2023 \\
Song's modified Barnard & 1497 & 447 & 0.2986 \\
Proposed improved Barnard & 2186 & 296 & 0.1354 \\
\hline
\end{tabular}

points indicate a higher degree of aggregation of feature points. We used a suppression radius of $r=3$ for calculating the entire image. The analysis results are shown in Table 2. After excluding the DoG and Song's modified Barnard algorithms, the algorithm developed in this study showed less trouble with excessive aggregation of detected feature points.

In summary, because the detection method of the DoG detector requires the construction of an image-scale space and a search for extreme values as feature points, it does not yield significant results when used on images with poor contrast. The Harris corner detector also has the same problem; therefore, these methods are not suitable for use on periapical X-ray images. The conventional Barnard detector uses areas with changes in gray-scale intensity as feature points, and a sufficient quantity of feature points can be extracted from such an image. However, this also causes feature points to be excessively aggregated. Although Song's modified Barnard algorithm can solve this problem, the feature points are not uniform and require manual adjustment of mask size, which is time-consuming. This method also cannot predict the detection quantity, resulting in too many or too few feature points. Through analysis, we found that our improved Barnard algorithm can greatly alleviate these problems.

\section{Conclusions}

A feature point detection algorithm was proposed for detecting representative feature points in periapical X-ray images. The proposed method can overcome nonuniform feature point distribution and can be used on low-contrast images. This algorithm was mainly the conventional Barnard algorithm improved by extracting distinctive points from images without obvious features. It could maintain the characteristics of the conventional Barnard algorithm: sufficient quantity and representativeness of feature points. During the screening of feature points, mask parameters were calculated using the image dimensions and expected quantity of feature points. Compared with Song's modified Barnard algorithm, the proposed method can greatly reduce the time needed for the manual adjustment of mask dimensions and improve the speed of the overall algorithm. The evaluation of the feature point distribution and analysis data showed that the proposed improved Barnard method is better superior to the other methods. The proposed algorithm is expected to be applicable to different types of images with poor contrast for detecting feature points. The proposed algorithm can not only identify representative and uniformly distributed feature points from periapical X-ray images but also can increase the accuracy of results when image registration is performed. 


\section{Acknowledgments}

This study was supported by the Ministry of Science and Technology, R.O.C. (grant No.: MOST 106-2218-E-239-001). The authors also gratefully acknowledge the financial support from the Cardinal Tien Hospital (grant Nos.: CTH-104-1-2A07 and CTH-104-1-2A06). We are thankful to our laboratory members Jia-Ling Ho, Shi-Xuan Wang, and Cheng-Jhong Lin, who provided expertise that greatly assisted the research.

\section{Author Contributions}

Conceptualization, Chia-Yen Lee and Chih-Chia Huang; Funding acquisition, Chia-Yen Lee and Chih-Chia Huang; Methodology, Liang-Hsin Wang; Project administration, Chia-Yen Lee; Resources, Chih-Chia Huang; Software, Liang-Hsin Wang; Supervision, Chia-Yen Lee and Chih-Chia Huang; Writing - original draft, Chia-Yen Lee; Writing - review \& editing, YuHsien Lin.

\section{Conflicts of Interest}

The authors declare no conflict of interest.

\section{References}

1 W. K. Murphy, G. E. Kaugars, W. K. Collett, and R. N. Dodds: Oral Surg. Oral Med. Oral Pathol. Oral Radiol. 74 (1991) 5.

2 J. Rud, J. O. Andreason, and J. M. Jensen: Int. J. Oral Maxillofac. Surg. 1 (1972) 4.

3 T. Lindeberg: Proc. 1996 CERN School of Computing (CERN, 1996) 27.

4 Y. Zhang and P. I. Rockett: IEEE Trans. Image Process. 15 (2006) 11.

5 K. Y. Li and Z. Dong: Proc. 2006 IEEE Int. Conf. Mechatronics and Automation (IEEE, 2006) 1503.

6 E. Rublee, V. Rabaud, K. Konolige, and G. Bradski: Proc. 2011 IEEE Int. Conf. Computer Vision (IEEE, 2011) 2564. https://doi.org/10.1109/ICCV.2011.6126544

7 F. Dellinger, J. Delon, Y. Gousseau, J. Michel, and F. Tupin: Proc. 2012 IEEE Int. Geoscience and Remote Sensing Symp. (IEEE, 2012) 3478. https://doi.org/10.1109/IGARSS.2012.6350671

8 C. Harris and M. Stephens: Proc. 1988 Alvey Vision Conf. (1988) 10.

9 G. Chenguang, L. Xianglong, Z. Linfeng, and L. Xiang: Proc. 2009 IITA 2009. Third Int. Symp. Intelligent Information Technology Application (IEEE, 2009) 49. https://doi.org/10.1109/IITA.2009.311

10 K. G. Derpanis: Proc. York University (2004).

11 H. Song, B. Li, and L. Zhang: Proc. 2006 IEEE Int. Conf. Intelligent Control and Automation (IEEE, 2006$) 49$.

12 Y. H. Tsai: Int. J. Comput. Electr. Autom. Control Inf. Eng. 3 (2009) 1.

13 S. T. Barnard and W. B. Thompson: IEEE Trans. Pattern Analysis and Machine Intelligence. PAMI-2 (1980) 4. https://doi.org/10.1109/TPAMI.1980.4767032 


\begin{abstract}
About the Authors
Chia-Yen Lee received her B.S. degree in biomedical engineering from Chung Yuan Christian University, Chung Li, Taiwan, in 2004, and M.S. and Ph.D. degrees in biomedical engineering from the National Taiwan University, Taipei, Taiwan, in 2005 and 2011, respectively. She is currently an assistant professor in the Department of Electrical Engineering, National United University, Miaoli, Taiwan. Her research interest includes biomedical image processing, artificial intelligence in medical imaging diagnostics, medical Imaging computeraided detection (CADe), computer-aided diagnosis (CADx), and medical device design and development.
\end{abstract}

Liang-Hsin Wang received her bachelor degree in electrical engineering from the National United University, Miaoli, Taiwan, in 2016. Her research interests include image processing, $\mathrm{X}$-ray image registration, and deep learning.

Yu-Hsien Lin received his B.S., M.S., and Ph.D. degrees in electronics engineering from National Chiao-Tung University, Hsinchu, Taiwan, R.O.C., in 2001, 2002, and 2006, respectively. His $\mathrm{Ph} . \mathrm{D}$. dissertation research focused on the engineering and physics of advanced memory devices (particularly nanocrystal-based ones). From 2006 to 2010, he was with the Taiwan Semiconductor Manufacturing Company (TSMC), Taiwan, as a Principle Engineer working on research and design for N40/N20 process integration and device performance improvement. In 2011, he joined the Department of Electronic Engineering, National United University, Miaoli, Taiwan, R.O.C., and currently he is full professor. His research interests include novel nonvolatile memory devices, high-k dielectric materials for CMOS devices, and poly-Si thinfilm transistors.

Chih-Chia Huang received his B.S. degree from Chung Shan Medical University, Taiwan, in 1997 and M.S. and Ph.D. degrees from the National Taiwan University College of Medicine., Taiwan, in 2007 and 2012, respectively. From 2013 to 2017, he was an assistant professor at Cardinal Tien Junior College of Healthcare and Management University, Taiwan. Since 2013, he has been the chief of the dental department at Cardinal Tien Hospital, An Kang area of Xindian district. His research interests are in oral radiology and VRF teeth. 